\title{
Argon metastables in HiPIMS: time-resolved tunable diode-laser diagnostics
}

C Vitelaru, Daniel Lundin, G D Stancu, N Brenning, J Bretagne and T Minea

\section{Linköping University Post Print}

N.B.: When citing this work, cite the original article.

Original Publication:

C Vitelaru, Daniel Lundin, G D Stancu, N Brenning, J Bretagne and T Minea, Argon metastables in HiPIMS: time-resolved tunable diode-laser diagnostics, 2012, Plasma sources science \& technology (Print), (21), 2, 025010.

http://dx.doi.org/10.1088/0963-0252/21/2/025010

Copyright: Institute of Physics

http://www.iop.org/

Postprint available at: Linköping University Electronic Press

http://urn.kb.se/resolve?urn=urn:nbn:se:liu:diva-77335 


\title{
Argon metastables in HiPIMS. Time-resolved tunable diode laser diagnostic
}

\section{Vitelaru ${ }^{1,2}$, D Lundin ${ }^{3}$, G D Stancu ${ }^{1}$, N Brenning ${ }^{4}$, J Bretagne $^{1}$, and T Minea ${ }^{1}$}

${ }^{1}$ Laboratoire de Physique des Gaz et Plasmas, UMR 8578 CNRS, Université Paris Sud-XI , 91405 Orsay Cedex, France

${ }^{2}$ Physics Department, Faculty of Physics Al I Cuza University, Bd. Carol No 11, Iasi, 700506, Romania

3 Plasma \& Coatings Physics Division, IFM-Materials Physics, Linköping University, SE-581 83 Linköping, Sweden

${ }^{4}$ Division of Space and Plasma Physics, School of Electrical Engineering, Royal Institute of Technology, SE-100 44 Stockholm, Sweden

E-mail: cvitelaru@plasma.uaic.ro

\begin{abstract}
Time resolved Tunable Diode-Laser Absorption Spectroscopy (TD-LAS) measurements were performed on the argon metastable $\left(\operatorname{Ar}^{\mathrm{m}}\right)$ level $3 s^{2} 3 p^{5}\left({ }^{2} \mathrm{P}^{\circ}{ }_{3 / 2}\right) 4 s$ excited at $801.478 \mathrm{~nm}$, in the dense plasma region in front of the magnetron target in a high power impulse magnetron sputtering (HiPIMS) discharge. From the Doppler profile the evolution of the temperature and density were derived during the pulse as well as during the plasma decay, i.e. in the afterglow. It is shown that the $\mathrm{Ar}^{\mathrm{m}}$ density sharply increases at the beginning of the discharge pulse, followed by a severe $\mathrm{Ar}^{\mathrm{m}}$ depletion along with increasing gas temperature around the peak of the HiPIMS discharge current. The strong dynamics of these parameters involve many elementary processes such as electron impact excitation, electron impact de-excitation and ionization of $\mathrm{Ar}^{\mathrm{m}}$, gas rarefaction, electron temperature increase at the end of the pulse, gas diffusion, etc. These phenomena are discussed with respect to several parameters: distance from the target, peak discharge current during the pulse, pulse length, as well as gas pressure.
\end{abstract}

PACS: $52.70 . \mathrm{Kz}, 52.70 . \mathrm{Nc}, 52.80 . \mathrm{Sm}$ 


\section{Introduction}

The field of ionized physical vapor deposition (IPVD) through high power impulse magnetron sputtering (HiPIMS) is continuously expanding, due to being a promising technique for improving common magnetron sputtering used in many industrial processes for thin film deposition [1]. One of the main advantages with this technique is the dramatic increase of the ionization degree of the metallic vapor, where it has been shown that the HiPIMS plasma generates large quantities of highly energetic ions [2] with, in some cases, a directed flux of charged species [3]. The mechanisms involved in how these energetic particles are transported and how they interact with the neutral gas background as well as the bulk plasma are not fully understood, but it is clear that they have a dramatic effect on thin film growth, such as densification and improved adhesion as well as improved film quality $[4,5]$.

The lower deposition rate for HiPIMS compared to conventional techniques for the same average power is often reported as a drawback, and is one of the most discussed topics in this field of research [1, 4, 6, 7, 8]. In recent publication by Samuelsson et al. [4] it was found that the rates are typically in the range of 30-85 \% compared to DC magnetron sputtering (DCMS) depending on source material. This highlights the need to understand the underlying physical mechanisms during the pulsed plasma regime. The working gas dynamics is one factor that is known to have a dramatic effect on the HiPIMS pulse characteristics [9] and thus affecting the rate and composition of the deposition flux. The reason for this is that during the sputtering process there is a certain probability that sputtered metal neutrals will collide with the background neutral gas. These collisions lead to gas heating followed by expansion (decrease in gas density in front of the cathode) in a process that is known as gas rarefaction. The depletion of gas has extensively been studied in magnetron discharges both experimentally and theoretically $[10,11,12,13,14,15]$ during the last three decades. In addition to gas rarefaction, there are also other effects leading to dissipation of neutral gas in front of the cathode, such as the presence of reflected, energetic neutrals, which have been accelerated as ions in the cathode sheath before being neutralized, reflected at the target surface and eventually involved in a collision event with the process gas. Furthermore, a direct loss of ionized working gas is also present, which involves gas ions being accelerated towards the cathode surface, then neutralized and still energetic enough to pass quickly through the near-cathode region [9]. The reduction of gas density would not play a major role if the refill process would be fast enough. However, this does not seem to be the case in all types of magnetron discharges, and gas rarefaction is believed to be particularly important in HiPIMS [16]. This has also been experimentally detected [9], where the commonly seen high-current transients cause a depletion of the working gas, and thereby a transition to a conventional DC magnetron sputtering-like high voltage, lower current regime, where the desired IPVD properties are lost. 
One of the main difficulties when investigating the dynamics of magnetron plasmas is the presence of the magnetic field trap, characterized by a highly inhomogeneous plasma with strong gradients of density, pressure, electric field etc. In addition, investigating the HiPIMS plasma requires time resolved measurements in order to describe the fast changes $(\sim \mu \mathrm{s})$ of different plasma parameters during the pulsed regime. Spectroscopic techniques are therefore suitable for the diagnostic of such plasmas, because they are non-intrusive and can be used to probe the plasma region very close to the cathode surface. Moreover, laser based techniques allow high spatial and spectral resolutions, and the measurements can be time resolved in order to follow fast phenomena $(<\mu s)[17]$.

Tunable Diode Laser Absorption Spectroscopy (TD-LAS) is a well established technique currently used to measure the density and temperature of absorbing species from high resolution absorption profiles. Although this technique is very efficient and easy to implement in continuous steady state discharges or time averaged measurements of plasma species $[18,19,20]$, the pulsed regime raises additional problems due to the fast time variations [21]. Time resolved measurements of atomic species have been performed by locking the laser to a fixed wavelength (usually the resonance wavelength $\lambda_{0}$ ) [22, 23]. Such time resolved measurements can easily give access to the general trend of the density evolution, but cannot give any information on the possible profile broadening. In the case of Doppler broadened profiles the information about the temperature variation is not available. Additionally, the time dependence of the entire absorption profile can be reconstructed by performing the measurements at different locked wavelengths [24]. One drawback of this method comes from the necessity to impose and easily control a very small $\Delta \lambda$ step (typically $0.1 \mathrm{pm}$ to have at least 20 points on the Doppler profile) during the whole measurement and over the desired spectral interval.

An absorption spectroscopy technique, which directly performs time resolved measurements in the afterglow of a pulsed discharge, has recently been proposed in [21]. This technique uses diode lasers in their regular scanning regime, at frequencies around $1 \mathrm{~Hz}$, and the precise synchronization between the laser scan and plasma pulses. This technique allows tracking the entire absorption profile during the plasma afterglow. New developments of the technique have permitted to make time resolved measurements also during the highly dynamic HiPIMS discharge.

The objective of this study is to investigate the neutral process gas dynamics during the HiPIMS discharge, since it is one of the key concepts towards understanding changes in deposition conditions. Through the use of TD-LAS it has been possible to resolve both in time and space the creation and loss of the argon metastable, $\mathrm{Ar}^{\mathrm{m}}$, which directly has given an insight into the development of the process gas temperature. Furthermore, the excitation and ionization as well as diffusion and decay processes operating during the HiPIMS discharge has been studied by following density variations of $\mathrm{Ar}^{\mathrm{m}}$ for different discharge conditions 


\section{Experimental details}

\subsection{Experimental setup}

A standard planar circular magnetron with a diameter of $10 \mathrm{~cm}$, equipped with a Ti $(99.9 \%)$ target mounted in a cylindrical vacuum chamber (height $0.50 \mathrm{~m}$, diameter $0.45 \mathrm{~m}$ ) was used in the present experiments. The chamber was pumped by a turbo-molecular pump to a residual pressure of about $1.3 \times 10^{-7} \mathrm{~Pa}$, after which Ar gas with a minimum purity of $99.9997 \%$ was let into the chamber reaching a pressure of 0.4 to $2.66 \mathrm{~Pa}$. Figure 1 shows a schematic of the vacuum chamber together with the optical setup. Unipolar pulses were applied between the cathode (target) and the chamber walls using a HiPIMS Sinex 3 (Chemfilt Ionsputtering AB, Sweden) power supply capable of delivering $2000 \mathrm{~V}$ and $1200 \mathrm{~A}$ peak values. In this work pulses up to approximately $500 \mathrm{~V}$ and $70 \mathrm{~A}$ (equivalent to a current density over the entire cathode surface of about $0.9 \mathrm{~A} \mathrm{~cm}^{-2}$ ) were used with a pulse duration of $200 \mu$ s and a repetition frequency of $50 \mathrm{~Hz}$, as seen in Figure 1.

Basic plasma parameters such as target voltage and target current as well as the absorption and emission signals were monitored and recorded on a LeCroy WaveSurfer 64Xs oscilloscope. A Tektronix P6015 high voltage probe measured the target voltage. The current was measured with a Chauvin Arnoux C160 current probe.

\subsection{Basic principles of TD-LAS}

The use of diode lasers in absorption measurement has been reviewed in [25, 26] for a large variety of atoms and different types of systems and devices. The use of these laser sources is based on the Lambert Beer law, describing the resonant absorption of laser radiation passing through an absorbent medium. The typical signals that have to be registered in this type of measurement are: the laser intensity without plasma $\left(I_{0}\right)$, the laser intensity after passing through the plasma $\left(I_{t}\right)$ (registered by the photodiode represented in Figure 1), and the laser intensity after passing through the Fabry Perot (FP) etalon (registered by the photodiode placed behind the FP etalon). The latter is needed to reconstruct the frequency scale, which is done by converting the time period between two successive maxima of the etalon into frequency (or wavelength), by knowing the etalons free spectral range (FSR).

Starting from the two registered curves, $I_{0}$ and $I_{t}$ the absorbance curve can be computed as $\ln \left(I_{0} / I_{t}\right)$, which in fact reconstructs the absorption profile $k(\lambda) L$, with $k(\lambda)$ being the absorption coefficient and $L$ the absorption length. This profile is then fitted with a Gaussian curve, which is characteristic to a thermalized distribution, and two parameters are extracted: the maximum absorption coefficient $k_{0}$ registered at $\lambda=\lambda_{0}$, and the full width at half maximum (FWHM ) of the absorption profile.

The absorption coefficient $k_{0}$ is proportional to the density of absorbing atoms, which depends on the transition characteristics $[27,28]$, and can be written on the simplified form: 


$$
k_{0}\left(\mathrm{~cm}^{-1}\right)=\frac{8.25 \cdot 10^{-13} \cdot f \cdot n\left(\mathrm{~cm}^{-3}\right)}{\delta \sigma_{D}\left(\mathrm{~cm}^{-1}\right)}
$$

where $f$ is the oscillator strength and $\delta \sigma_{D}=\Delta \lambda_{F W H M} / \lambda^{2}$ the Doppler broadening. Starting from this relation one can express the atomic density $n$ as a function of constants related to the transition and the measured parameters $k_{0}$ and $\Delta \lambda_{F W H M}$.

$$
n\left(\mathrm{~cm}^{-3}\right)=\frac{k_{0} \cdot \Delta \lambda_{F W H M}}{8.25 \cdot 10^{-13} \cdot f \cdot \lambda^{2}}
$$

The main line broadening mechanism, in the absence of high magnetic or electric fields, is the Doppler broadening, caused by the movement of atoms towards the laser source or in the opposite direction. In the case of a Maxwell velocity distribution function the line shape results in Gaussian profile. The value of the Doppler broadening depends on the temperature and mass of absorbing atoms [29] and can be written under the simplified form:

$$
\frac{\Delta \lambda_{F W H M}}{\lambda^{2}}=\frac{7.16 \cdot 10^{-7}}{\lambda(\mathrm{cm})} \sqrt{\frac{T(K)}{M(a m u)}}
$$

The atomic temperature can therefore be expressed as:

$$
T_{T i}(K)=M\left(\frac{\Delta \lambda_{F W H M}}{\lambda} \frac{1}{7.16 \cdot 10^{-7}}\right)^{2}
$$

\subsection{Principles of time resolved TD-LAS}

The rapid changes during the pulsed plasma make it necessary to develop time resolved diagnostic methods. The TD-LAS technique is usually employed for stationary plasmas [30]or time averaged measurements in pulsed plasmas [31], but has been adapted in various ways to perform time resolved measurements $[21,22,24]$. The method proposed in this paper uses the diode laser in its regular scanning mode, at very low scanning frequency (less than $0.2 \mathrm{~Hz}$ ).

The schematic diagram of devices and the synchronization signals used in this experiment are presented in Figure 3. The power supply is working independently of any external trigger, at a fixed frequency of $50 \mathrm{~Hz}$ (plasma pulses are equally spaced by $20 \mathrm{~ms}$ off-time periods). The laser diode scan frequency is fixed at much lower value, so that during one half period of the wavelength scan a few hundreds of plasma pulses may occur ( 1000 pulses in our case). In this way the wavelength variation during one plasma pulse $(200 \mu \mathrm{s})$ is very small $(<0.001 \mathrm{pm})$, and can be considered constant. The beginning of a diode laser ramp (change of slope) is used as a trigger to initiate the data acquisition on the oscilloscope, and the sequence of data is only registered during one half period diode laser scan. In order to perform these measurements it is necessary to use a high frequency sampling oscilloscope (450MHz bandwidth, 2Gs/s sample rate in our case), which permits to register sequences of data, 
triggered by an external signal which is in our case the repetition frequency of the pulsed plasma. By using this technique absorption time traces of typically 100 pulses, like the one shown in the inset of Figure 3, are registered. Let us note that from the 1000 plasma pulses occurring during one half of diode laser scan period we only register each tenth pulse, resulting in 100 equally spaced pulses registered at the end of the acquisition stage.

Simultaneously with the absorption signal, the signal coming from the FP etalon is registered on a different channel of the oscilloscope. In the acquired time window the amplitude of this signal is practically constant, the wavelength and the laser power variation being negligible. In fact, the rate of laser wavelength variation is $0.7 \mathrm{pm} / \mathrm{s}$, so during one period of pulsed plasma (20 ms) the wavelength variation is $0.014 \mathrm{pm}$ and it is only $0.7^{*} 10^{-3} \mathrm{pm}$ in the registered time window of $1 \mathrm{~ms}$. Let us note that the FWHM of the Doppler broadened line profile is about $2 \mathrm{pm}$ at room temperature.

The reconstruction of the FP etalon signal is made by representing the average value of FP intensity over the acquisition time window (1 ms) as function of pulse number. The resulting plot is represented in Figure 4 with black squares. The etalon is characterized by a free spectral range (FSR) of $1 \mathrm{GHz}$, which means that the peaks in the FP signal are equally spaced with $1 \mathrm{GHz}$ frequency shift between them. Using this information one can transform the "pulse No" scale into a frequency scale, which is represented in the upper part of Figure 4. By doing so, each point in the graph, meaning each registered plasma pulse, is associated with a frequency (or wavelength) shift. Let us note that the origin of the frequency scale was chosen so that it corresponds to the HiPIMS pulse characterized by the maximum absorption signal, so that the laser wavelength (or frequency) equals the transition wavelength (or frequency) $v=v_{0}$.

In the same graph the evolution of laser intensity without plasma $\left(I_{0}\right)$ is also represented during one half period of diode laser scan. Each point in this plot is resulting from the averaging of values registered on the absorption signal, but only for the period of time before the ignition of the pulse (roughly the first $100 \mu$ s in the absorption vs time plot in Figure 3). For this time period we assume that the level of $\mathrm{Ar}^{\mathrm{m}}$ is insignificant (background level). These values will serve as $I_{0}$ (laser intensity without plasma), in the computation of absorbance.

At the end of the data acquisition stage, around 100 time traces will be recorded, each one representing the time variation of laser intensity during $1 \mathrm{~ms}$ around the plasma pulse, at a fixed frequency shift corresponding to the points in Figure 4. These time traces are introduced as lines into a matrix, which is then represented as a $2 \mathrm{D}$ contour plot like in Figure 5. The time variation can be followed on the horizontal axis, whereas the frequency shift can be followed on the vertical axis.

This image offers a general view on the time variation of the absorption profile. The horizontal cut at $\Delta v=0$ in Figure 5 represents the time variation of absorbance at a fixed wavelength, given in the top inset in Figure 5. On the other hand, the vertical cut in the same figure represent the absorption profile at a fixed moment of time: a profile which is constituted by $\sim 100$ points corresponding to the same number of registered plasma pulses. The right hand side inset in Figure 5 
represents such an absorption profile, obtained at $30 \mu$ s after plasma ignition. In the same figure, the Gaussian fit of the profile is also represented, showing good agreement with the experimental points.

Assuming the Doppler effect as the main broadening mechanism affecting the line profile, the gas temperature $\left(\mathrm{Ar}^{\mathrm{m}}\right)$ can be deduced from the FWHM of the absorbance profile. Hence, the data acquired and used for the 2D representation in Figure 5 allows computing the density and temperature of $\mathrm{Ar}^{\mathrm{m}}$ in virtually any instant of time during the plasma pulse, with a time step of $0.1 \mu \mathrm{s}$. Since the expected time constant of involved processes is at least one order higher, we choose to compute the density and temperature with a bigger time step. In this way the amount of processed data can be significantly reduced, while preserving a good time resolution in the final results. The chosen time step is $2.5 \mu$ s during the plasma pulse and $5 \mu \mathrm{s}$ in the after-glow period, the dynamic being slower in that later interval. The experimental results given below is therefore represented as time traces of density and temperature variation during the plasma pulse $(200 \mu \mathrm{s})$ as well as during the beginning of the afterglow (200 $\mu$ s, which is in the next section seen to be long enough to study the relevant processes).

\section{Results and discussion}

\subsection{General features of the gas dynamics}

An example of a time-resolved $\mathrm{Ar}^{\mathrm{m}}$ density-temperature profile taken at $z=1 \mathrm{~cm}$ above the target race track at $p_{A r}=1.33 \mathrm{~Pa}$ is given in Figure 6. The labels $\mathrm{t}_{1}-\mathrm{t}_{4}$ (also marked as color-coded regions) indicate the different time intervals where the most striking features were found. These features were seen in all measurements, although the magnitude of the individual peaks as well as exact position in time varied depending on the discharge conditions and are addressed in detail in the following subsections. Table 1 summarizes the different features for the chosen time intervals.

Following the time-evolution of $n_{A r^{m}}$ and $T_{A r^{m}}$ for the different time intervals $\mathrm{t}_{1}-\mathrm{t}_{4}$ it is first seen that during the onset of the HiPIMS discharge pulse $\left(\mathrm{t}_{1}\right)$ the rise of $n_{A r^{m}}$ is considerably faster than the rise of the discharge current itself. From Langmuir probe measurements on these type of discharges [32] it is known that there is a strong increase in $n_{e}$ as well as $T_{e}$ during the initial part of the HiPIMS pulse. Thus, the probability for electron impact excitation of argon, $\mathrm{e}+\mathrm{Ar} \rightarrow \mathrm{Ar}^{\mathrm{m}}+\mathrm{e}$, is greatly increased by the increasing population of energetic electrons. Furthermore, Poolcharuansin et al. have detected a short burst of hot electrons (tens of $\mathrm{eV}$ ) only within the first $10 \mu$ s of the HiPIMS pulse [33], which would explain the great increase in the number of $\operatorname{Ar}^{\mathrm{m}}$ present during $\mathrm{t}_{1}$. The temperature of $\mathrm{Ar}^{\mathrm{m}}$ also starts to increase, which is likely due to collisions of Ar with the steadily increasing amount of sputtered metal neutrals having an energy that can go up to about 5-15 eV [18]. 
We have to note that $\mathrm{Ar}^{\mathrm{m}}$ temperatures of $600-900 \mathrm{~K}$ have also been previously obtained [34] in DC magnetrons, so the temperature increase in this interval is within the limits of a high power DC magnetron discharge (600 Volts and up to $5 \mathrm{~A})$.

Table 1. A summary of the features seen in the HiPIMS discharge current as well as the general trends for a typical TD-LAS measurement for four different stages of the HiPIMS discharge. The time intervals $t_{1}-t_{4}$ are shown in Figure 6.

\begin{tabular}{|c|c|c|}
\hline $\begin{array}{l}\text { Time } \\
\text { interval }\end{array}$ & $\begin{array}{l}\text { Time after pulse } \\
\text { initiation }[\mu \mathrm{s}]\end{array}$ & Feature \\
\hline $\mathrm{t}_{1}$ & $0-25$ & $\begin{array}{l}\text { Early stage of the discharge pulse, discharge current is very } \\
\text { low }\left(I_{D}<10 \mathrm{~A}\right) \text {. A fast increase of } n_{A r^{m}} \text {, which peaks at the } \\
\text { end of this period as well as an increase of } T_{A r^{m}} \text {. }\end{array}$ \\
\hline$t_{2}$ & $25-80$ & $\begin{array}{l}\text { Strong increase and eventually peak of the discharge current } \\
\left(I_{D}=40 \mathrm{~A}\right) \text {. Considerable decrease of } n_{A r^{m}} \text {, and a } \\
\text { stabilization of } T_{A r^{m}} \text {. }\end{array}$ \\
\hline$t_{3}$ & $80-200$ & $\begin{array}{l}\text { Decay of discharge current until pulse-off. New increase of } \\
n_{A r^{m}} \text { and a second increase of } T_{A r^{m}} \text {. }\end{array}$ \\
\hline $\mathrm{t}_{4}$ & $200-400$ & $\begin{array}{l}\text { Plasma afterglow, strong decay of both } n_{A r^{m}} \text { and } T_{A r^{m}} \text {. No } \\
\text { discharge current. }\end{array}$ \\
\hline
\end{tabular}

It is here assumed that $T_{A r^{m}} \approx T_{A r}$ since $\mathrm{Ar}^{\mathrm{m}}$ are produced from Ar and no great additional heating of the $\mathrm{Ar}^{\mathrm{m}}$ is expected, which is why $T_{A r}$ will be used from here on. This assumption is certainly reasonable around $2 \mathrm{~Pa}$ and above, where one expects thermal equilibrium between $\mathrm{Ar}$ and $\mathrm{Ar}^{\mathrm{m}}$. In the same time we should note that due to the investigated time scale on the order of $\mu$ s or tens of $\mu \mathrm{s}$ it is possible to have transient regimes for which the complete equilibrium is not acquired. Nevertheless, the width of the absorption profile, converted into temperature, gives information on the thermal energy gained by the Ar atoms during the HiPIMS process.

As the pulse progresses $\left(t_{2}\right)$ the metal atom and argon metastable densities build up and loss processes, such as Penning ionization $\mathrm{Ar}^{\mathrm{m}}+\mathrm{Ti} \rightarrow \mathrm{Ti}^{+}+\mathrm{e}+\mathrm{Ar}$, electron impact ionization of $A r^{m}$, $\mathrm{e}+A r^{m} \rightarrow A r^{+}+2 e$, as well as electron impact deexcitation, $\mathrm{Ar}^{\mathrm{m}} \rightarrow \mathrm{Ar}$, start to compete with the generation of $\mathrm{Ar}^{\mathrm{m}}$ and reduce the growth rate of $n_{A r^{m}}$. A balance between production and loss is eventually established, probably around the time of the first $\mathrm{Ar}^{\mathrm{m}}$ density peak, at an equilibrium value 
of the ratio $n_{A r^{m}} / n_{A r}$. Another important factor is electron impact excitation and ionization of sputtered $\mathrm{Ti}$ atoms, $\mathrm{e}+\mathrm{Ti} \rightarrow \mathrm{Ti}^{+}+2 \mathrm{e}$, which effectively cool down the electrons in the plasma, since the ionization potential of $\mathrm{Ti}(6.82 \mathrm{eV})$ is well below the one for $\operatorname{Ar}(15.76 \mathrm{eV})$. In summary, the peak of $n_{A r^{m}}$ is likely to be determined by several processes, such as the abundance of low energy electrons (a few $\mathrm{eV}$ ), which can activate a very efficient loss channel through electron impact ionization of $\mathrm{Ar}^{\mathrm{m}}$. In addition, the character of the entire electron energy distribution function (EEDF) will affect the generation and loss of $\mathrm{Ar}^{\mathrm{m}}$ through the various mechanisms mentioned above. Also other loss channels for $\mathrm{Ar}^{\mathrm{m}}$ not governed by electrons, such as Penning ionization, play a role. Until more detailed studies of the effects of the different processes can be performed using for example plasma modeling, it can so far only be concluded that the plasma conditions at this point during the pulse are such that the above described generation processes are balanced by loss mechanisms.

As the peak of the HiPIMS discharge current is approached $\left(t_{2}-t_{3}\right)$ there is a strong decrease of $n_{A r^{m}}$ while $T_{A r}$ continues to increase. In addition to the previously discussed processes the severe reduction of $\mathrm{Ar}^{\mathrm{m}}$ can be associated with a strong depletion of neutral Ar, which is known to take place in this type of discharge [9]. Here it is likely that reduction of neutral argon is dominated by electron impact ionization due to the much higher plasma densities during the peak of the HiPIMS discharge current as compared to for example DCMS [35]. Thermal expansion, where the heating is due to momentum transfer in collisions between the background gas and sputter-ejected target atoms as well as reflected sputtering gas atoms is also contributing [16], but the contribution of each process to the general behavior has not been quantified here.

During the decay of the discharge current $\left(\mathrm{t}_{3}\right)$ the plasma density decreases causing a reduction of Ar ionization and thereby reduced sputtering (i.e. decreasing metal flux). The overall effect on the gas dynamics is a decrease of the gas heating (fewer collisions) and ultimately a reduction of the gas depletion. At the same time the gas loss mechanisms close to the target are counteracted by gas refill, which for reference has a time constant of 50-100 $\mu$ s for refill with the thermal speed of Ar at room temperature and over $0.02 \mathrm{~m}$. The minimum in $n_{A r^{m}}$ is met where the gas refill balances the gas loss mechanisms. As the discharge current decays the gas refill will start to dominate, which is the expected dominant process explaining the steady increase of $n_{A r^{m}}$ during $t_{3}$.

At $t=200 \mu$ s the HiPIMS pulse is switched off and the plasma goes into an afterglow $\left(\mathrm{t}_{4}\right)$, which is characterized by a decay in both density and temperature of $\mathrm{Ar}^{\mathrm{m}}$ as the plasma species are lost through recombination and diffusion towards the chamber wall. Note that during $\mathrm{t}_{4}$ the decay of $n_{A r^{m}}$ is characterized by an initial fast decrease followed by a more moderate reduction, which is further 
discussed in section 3.5. In summary, it is seen that several factors, such as $n_{e}$, EEDF, ionization, gas depletion, gas refill, and metal flux of sputtered atoms influence the behavior of $n_{A r^{m}}$ and $T_{A r}$.

\subsection{Effects of HiPIMS peak current}

The time-evolution of $n_{A r^{m}}$ and $T_{A r}$ for four different applied peak discharge currents $\left(I_{D, p e a k}=0.5 \mathrm{~A}\right.$, $10 \mathrm{~A}, 40 \mathrm{~A}$, and $70 \mathrm{~A}$ ) have been studied and are given in Figure 7. A faster increase of $n_{A r^{m}}$ for larger currents can be seen, which is mainly due to a faster rise of the HiPIMS discharge current, which is correlated with the faster increase of the electron density. When studying the two cases with the lowest peak currents it can also be concluded that the increase in $n_{A r^{m}}$ rather closely follows the behavior of the discharge current itself. In addition, there does not seem to be a decrease in $n_{A r^{m}}$ during the peak of the HiPIMS pulse. We believe that the $\mathrm{Ar}^{\mathrm{m}}$ density evolution is dependent on the amount of electrons available: if a certain critical electron density is reached (as in the case of high peak discharge current) the loss processes of $\mathrm{Ar}^{\mathrm{m}}$ will become dominant and $n_{A r^{m}}$ starts to decrease. In addition, the low current discharges resemble DCMS discharges, where the electron density is 2-3 orders of magnitude lower [36], which also means that the loss of neutral Ar is less of a problem. This is particularly true for the $0.5 \mathrm{~A}$ case, whereas the $10 \mathrm{~A}$ case shows a slight decrease of $n_{A r^{m}}$ at $t>75$ $\mu \mathrm{s}$, which is likely due to increased ionization of Ar by energetic electrons (i.e. reduction of $\mathrm{Ar}^{\mathrm{m}}$ ) with increasing discharge current densities.

Moreover, in the case of lower applied peak current the first $n_{A r^{m}}$ peak appears later in time. Let us remember that $n_{A r^{m}}$ is due to the balance between the production and loss processes. Following the idea that the maxima of $n_{A r^{m}}$ is reached for the same critical $n_{e}$. The loss mechanisms are however generally slower, so that for very short rise times some loss channels do not affect $n_{A r^{m}}$ to any greater extent leading to a higher $n_{A r^{m}}$ peak. Hence, the faster the rise of $n_{e}$, the higher the first peak of $n_{A r^{m}}$.

For higher currents, the first peak is followed by a much stronger depletion of $\mathrm{Ar}^{\mathrm{m}}$, which is a result of the increased power density (i.e. electron density) causing severe reduction of Ar as previously discussed. Also, $T_{A r}$ is generally increased for increased applied discharge current, which is in line with the increased sputtering of metal particles resulting in more Ar-Ti collisions and thus increased momentum transfer to the neutral gas background resulting in increased $T_{A r}$ (projectile effect). The delay in time (about $100 \mu$ s) until the temperature sharply increases for the high discharge current cases can be understood based on the time it takes to ionize Ar in order to sputter Ti plus the flight time of Ti out into the bulk plasma. 


\subsection{Process gas pressure effects}

The time-evolution of $n_{A r^{m}}$ and $T_{A r}$ for different Ar process pressures ( $p=0.67 \mathrm{~Pa}, 1.33 \mathrm{~Pa}, 2.00 \mathrm{~Pa}$ and 2.67 Pa) are shown in Figure 8. In general, the discharge current characteristics were similar in all cases (Figure 8, top) and only small differences in the temperature-density plots were detected. A higher initial increase of $n_{A r^{m}}$ for increased pressure can be seen. As can be seen in Figure 9 this is mainly a result of the increase in source particles for $\operatorname{Ar}^{\mathrm{m}}$ (i.e. increased $n_{A r}$ ), since the rise of the discharge current, and thereby the supply of electrons, do not vary significantly between the different operating pressures. Furthermore, the second peak of $n_{A r^{m}}$ at the end of the pulse indicates a smaller increase in density for increased process pressure. It could be due to the faster decrease of the discharge current for the $p=0.67 \mathrm{~Pa}$ case. For the other cases only minor differences can be observed. $\mathrm{The}^{\mathrm{m}}{ }^{\mathrm{m}}$ temperature evolution does not differ much when varying the process pressure. This behavior is consistent with the very small changes of the current pulse with the pressure. Indeed, the sputtering is related to the flux of ions to the cathode $\left(n_{e} \quad n_{i}\right)$ and their energy should slightly change. For all four pressure values used the sheath can be assumed collisionless.

\subsection{Spatial-dependent effects}

The time evolution of $n_{A r^{m}}$ and $T_{A r}$ depending on the distance to the target surface was recorded and is shown in Figure 10. The region closest to the target $(z=1 \mathrm{~cm})$ gives rise to the most distinct features of both the density and temperature, such as: 1) the strongest initial increase of $n_{A r^{m}}, 2$ ) severe gas density reduction during the current peak of the HiPIMS pulse as well as 3) the highest gas temperature at the end of the pulse and during the $t_{2}$ period. For the measurements performed further away from the target, these features were smoothened out and their amplitude decreased. There are several reasons for this behavior: further away from the cathode the energetic secondary electrons have lost much of their energy through collisions, which is why the previously seen sharp increase in $n_{A r^{m}}$ is not detected in Figure 10b-c, although the trend is there. The same holds true for the sputtered metal particles, which traverse the intense plasma region and undergo collisions on their way out into the chamber. Collisional gas heating is thereby expected to decrease, resulting in a lower $T_{A r}$, which is also seen in Figure 10.

\subsection{Effects of HiPIMS pulse width}

Last, the effects of changing the discharge pulse width while keeping all other parameters constant were investigated by varying the pulse-on time between 80 - $200 \mu$ s in steps of $20 \mu$ s. As it can be seen in the top and middle panel of Figure 11 the pulse characteristics are identical in all cases up till the 
point of terminating the pulse, indicating that the plasma conditions underwent the same initial evolution. The only difference is that the discharge current at the point of terminating the HiPIMS pulse was substantially different between the seven cases investigated, ranging from about $38 \mathrm{~A}$ for the $80 \mu$ s pulse down to about 3 A for the $200 \mu$ s pulse. From the recorded absorbance intensity measuring the relative density of $\mathrm{Ar}^{\mathrm{m}}$ several striking features can be seen: 1) the first $n_{A r^{m}}$ peak increases with increasing pulse width, 2) the previously detected second density peak gets higher as the pulse width increases, 3 ) there is a faster density decay as the pulse width is decreased, and 4) the decay of $n_{A r^{m}}$ is characterized by an initial fast decrease followed by a considerably slower reduction in density.

Very little is known about the afterglow regime of the HiPIMS discharge, but from Langmuir probe measurements [33] it is found that a weak plasma can be sustained for several microseconds (> $10 \mu \mathrm{s})$ after pulse-off. It is therefore possible that the increased amplitude of the first peak of $n_{A r^{m}}$ as the pulse width increases is related to the fact that the plasma decays slower and thus the bulk plasma will contain more $\mathrm{Ar}^{\mathrm{m}}$ at the beginning of the next HiPIMS pulse. The density of the remaining $\mathrm{Ar}^{\mathrm{m}}$ atoms might be a few orders of magnitude lower than the one during the pulse, being thus below the detection limit of the absorption technique. One can see that the density of $\mathrm{Ar}^{\mathrm{m}}$ at $\mathrm{t}=400 \mu$ s is higher with increasing pulse width, showing that there is a stronger chance that they will survive longer in the afterglow phase. Furthermore, the increasing second $n_{A r^{m}}$ peak with increasing pulse width can be understood, since the decay phase of the HiPIMS discharge current is prolonged and thereby sustaining the discharge and electron impact excitation in the phase of the pulse where gas refill starts to dominate.

Finally, the change in decay rate of $n_{A r^{m}}$ at pulse-off is likely a combination of two different processes: initially the fast process of electron impact deexcitation is predominant, while on longer timescales the much slower process of diffusion of $\mathrm{Ar}^{\mathrm{m}}$ prevails. The differences in rate seen for the different pulse widths are related to the difference in discharge current at the time of pulse-off. From previous investigations on the plasma conditions between the peak of the discharge current and the end of the HiPIMS pulse during similar discharges it is known that $n_{e}$ decreases by almost an order of magnitude, while $T_{e}$ does not change significantly [37]. This means that the plasma conditions at pulse-off will be different depending on pulse-width, where the short pulses represent the peak of an ordinary HiPIMS pulse characterized by strong sputtering of metal, high ionization rate, and severe rarefaction of Ar, while the long pulses represent the decay phase with decreasing ionization rate, reduced gas depletion and strong gas refill. It is therefore expected that the loss mechanisms of $\mathrm{Ar}^{\mathrm{m}}$ are dominant during the short pulses, which explains the faster decay rate of $n_{A r^{m}}$. 


\section{Conclusions}

Time resolved TD-LAS has proven to be a highly useful technique for monitoring the neutral gas dynamics, where the first results on the $\mathrm{Ar}^{\mathrm{m}}$ density and temperature evolution in a HiPIMS discharge have been generated. It is shown that there is a substantial increase of $n_{A r^{m}}$ at the beginning of the discharge pulse, which is followed by severe $\mathrm{Ar}^{\mathrm{m}}$ depletion along with increasing gas temperature around the peak of the HiPIMS discharge current. These trends differ substantially from a discharge running DCMS-equivalent currents, where the reported gas depletion for the high-current pulses

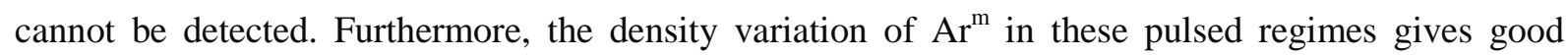
insight into the ionization and excitation processes, where strong electron impact ionization reduces the amount of neutral Ar available. The results are valuable input into ongoing HiPIMS plasma modeling, which can more accurately predict the dominating creation and loss processes of each species present in the discharge with the ultimate goal of optimizing any type of HiPIMS process with respect to the desired thin film properties.

\section{Acknowledgements}

This work was partly supported by the Romanian Grant 540/2009 within the IDEI program of the PNCDI, the Swedish Research Council, the Swedish Foundation for Strategic Research and the European Collaboration in Science and Technology (COST Action: MP0804). 


\section{Figures}

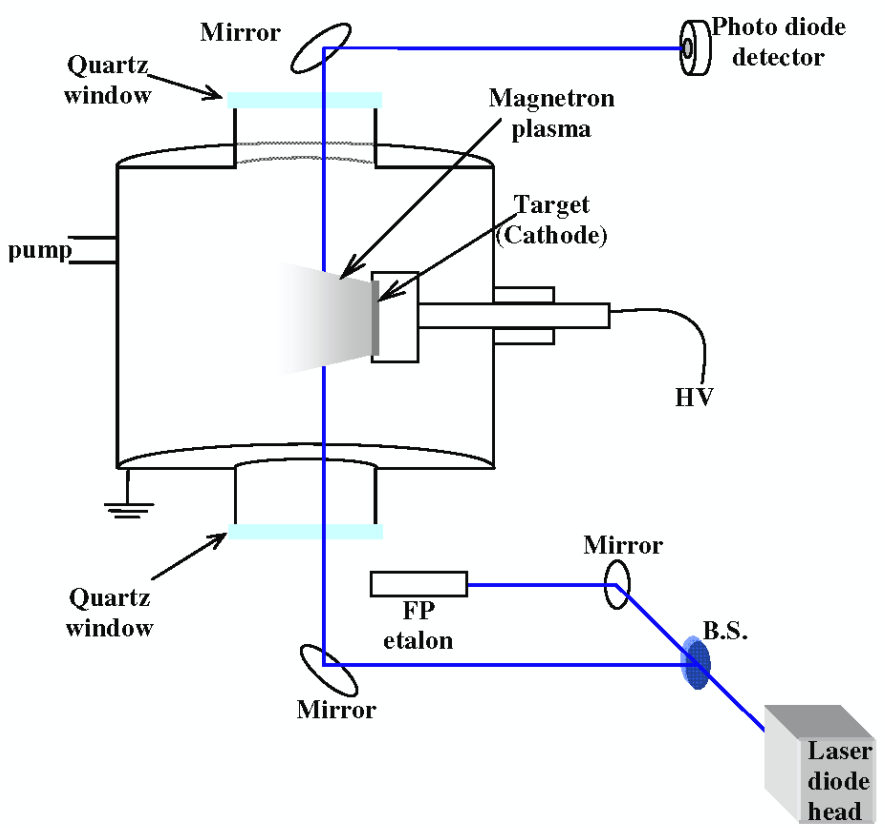

Figure 1. Schematic of the deposition system used in the experiments along with the optical arrangement of the TD-LAS for $\mathrm{Ar}^{\mathrm{m}}$ diagnostics in HiPIMS.

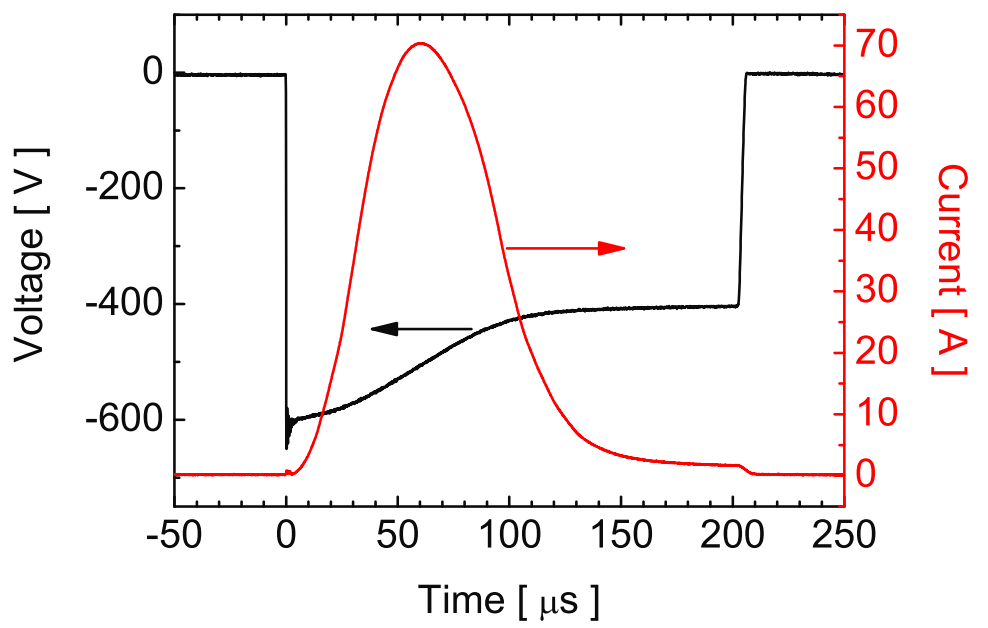

Figure 2. Typical shape of the HiPIMS current and voltage during a discharge pulse. 


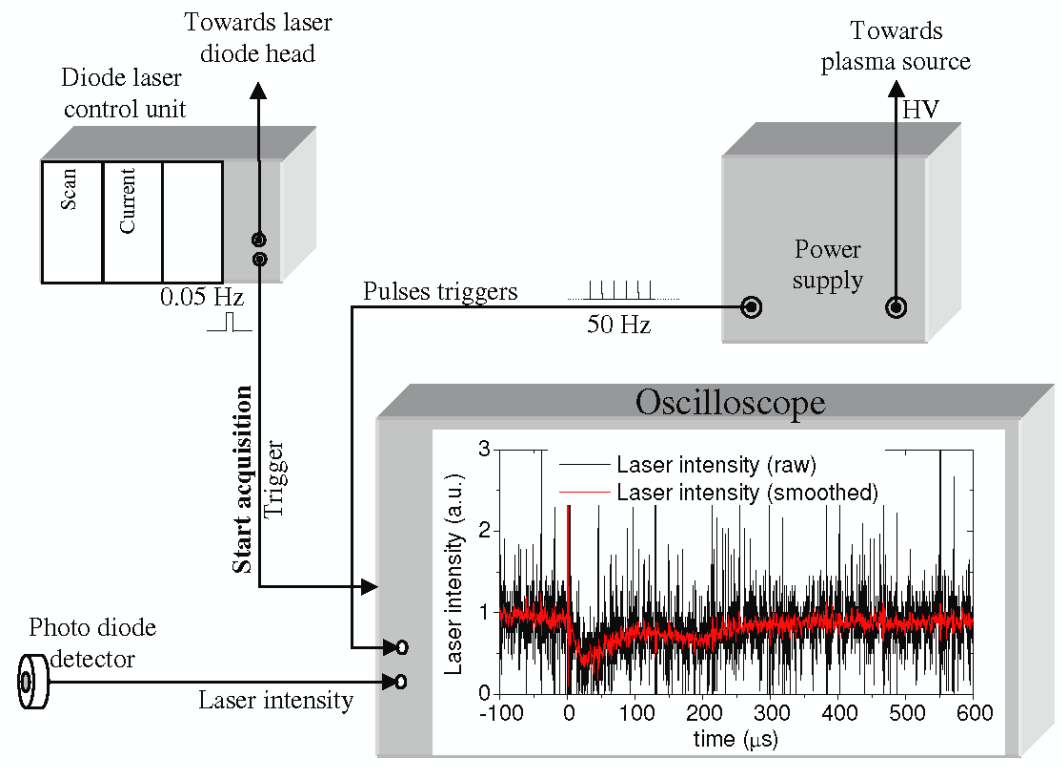

Figure 3. Schematical diagram of the trigger synchronization for time resolved TD-LAS in HiPIMS.

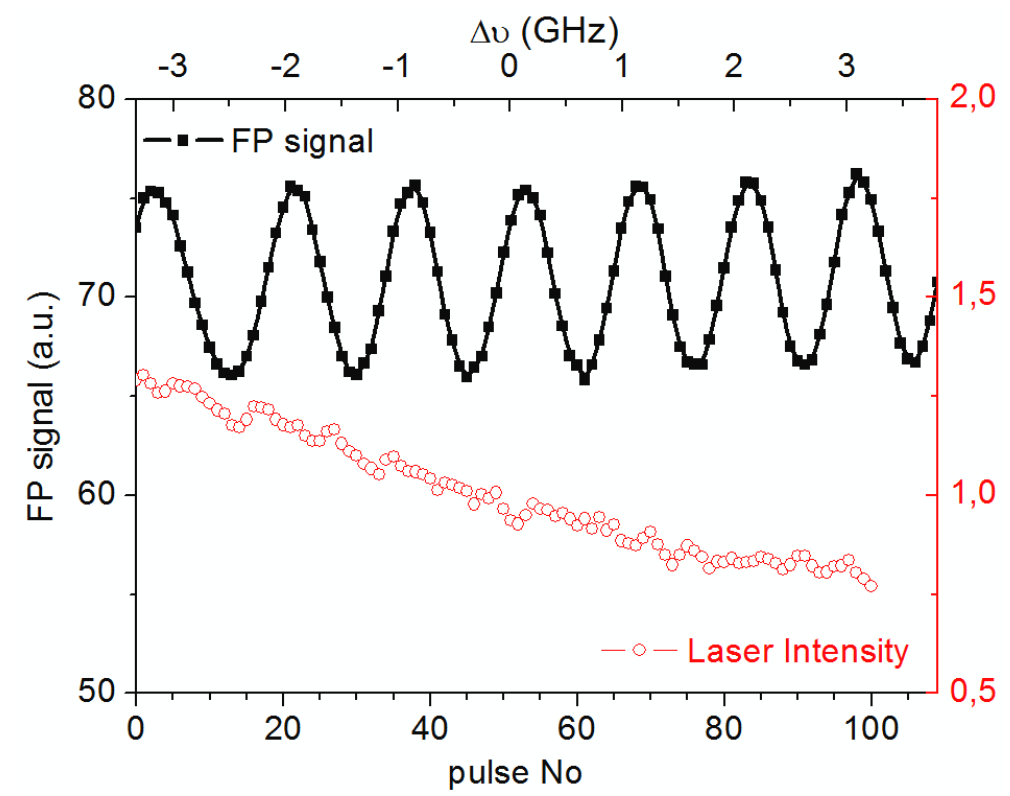

Figure 4. Typical FP etalon spectra and laser intensity without plasma $I_{0}$ during one ramp of the diode laser scan. 


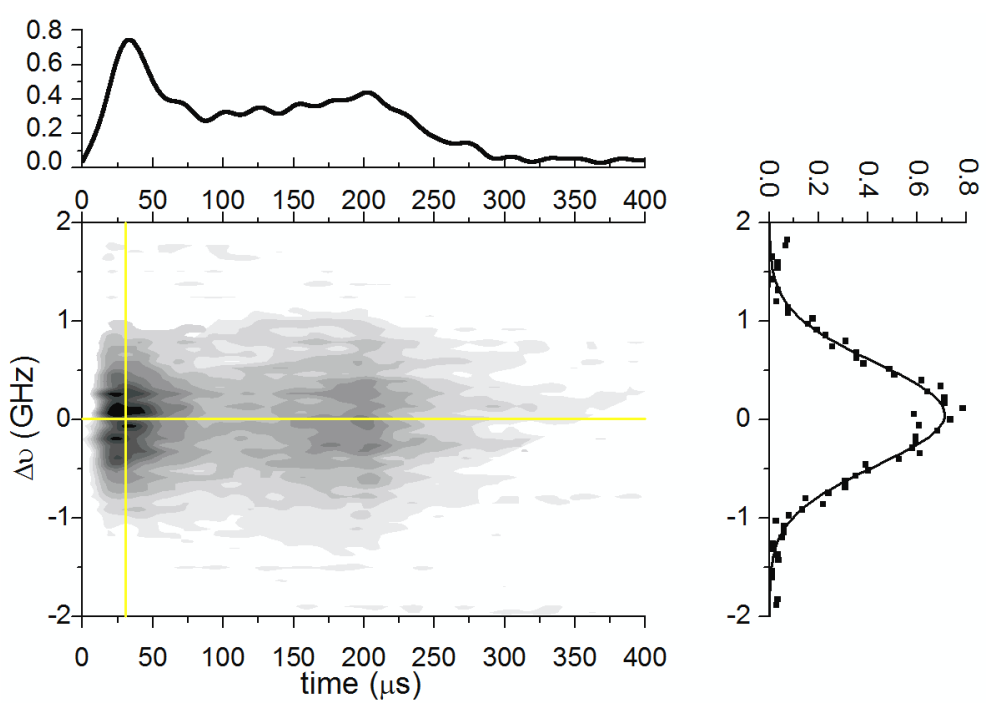

Figure 5. Typical result of time/frequency variation of absorbance signal, during the discharge pulse (200 $\mu \mathrm{s})$ and $200 \mu \mathrm{s}$ after the pulse suppression, i.e. the beginning of the afterglow. Plasma parameters are: $I_{D}=$ $40 \mathrm{~A}, p=2.0 \mathrm{~Pa}$, and $z=1 \mathrm{~cm}$.

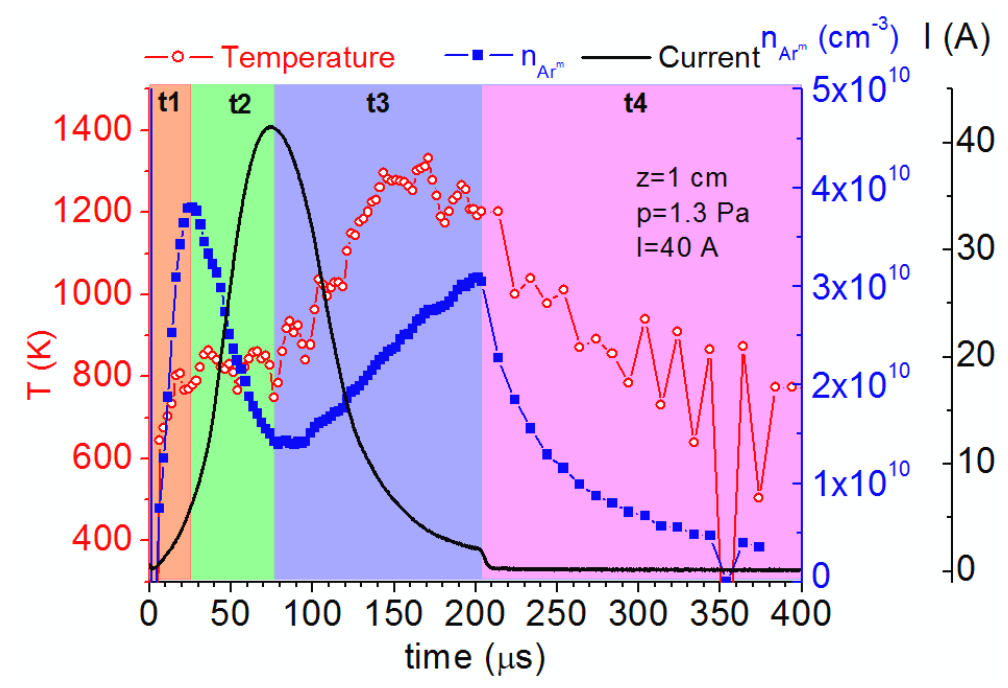

Figure 6. Typical results of the time resolved TD-LAS in HiPIMS. The full black curve displays the HiPIMS discharge current $I_{D}(t)$, the red curve with circles is the temperature of the $\mathrm{Ar}^{\mathrm{m}}$ and the blue curves with squares represents the $\mathrm{Ar}^{\mathrm{m}}$ density. 

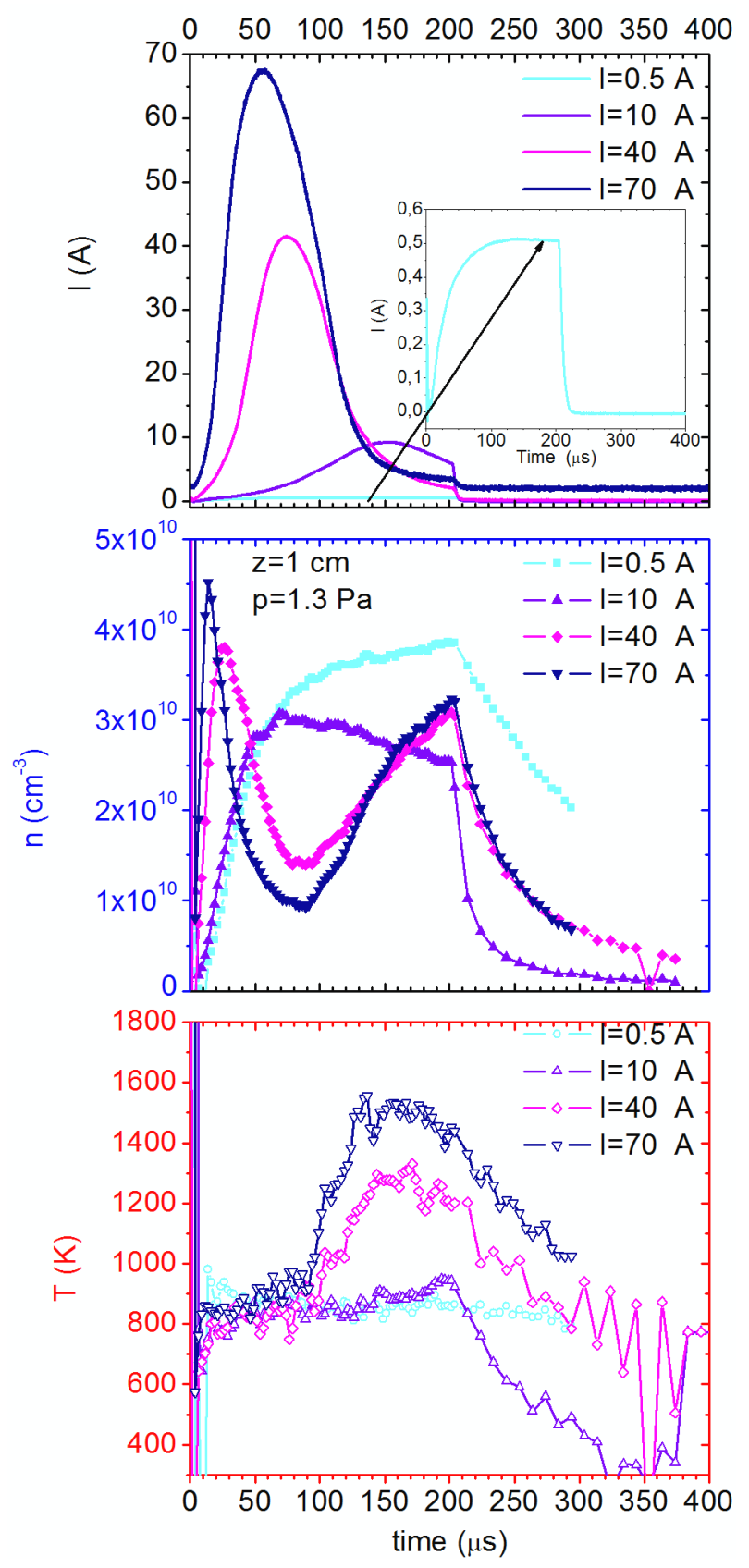

Figure 7. Time evolution of (top) HiPIMS current; (middle) $\mathrm{Ar}^{\mathrm{m}}$ density; (bottom) $\mathrm{Ar}^{\mathrm{m}}$ temperature at $1.33 \mathrm{~Pa}$, measured at $1 \mathrm{~cm}$ in front of the target for different maximum peak currents $(0.5,10,40$, and 70 A). 

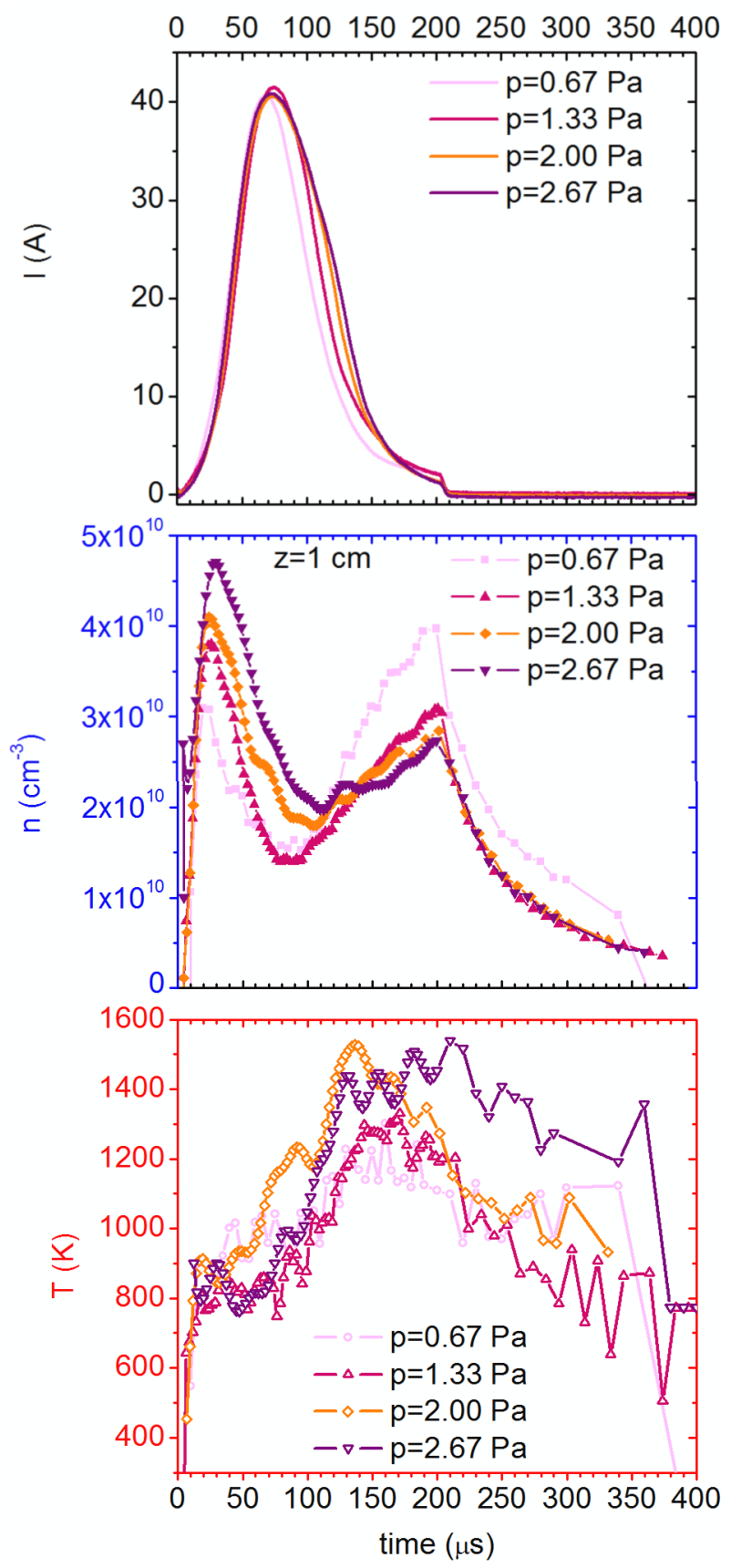

Figure 8. Time evolution of (top) HiPIMS current; (middle) $A r^{\mathrm{m}}$ density; (bottom) $\mathrm{Ar}^{\mathrm{m}}$ temperature for 40 A peak current, measured at $1 \mathrm{~cm}$ in front of the target for different pressures $(0.67,1.33,2.00$, and 2.67 Pa). 


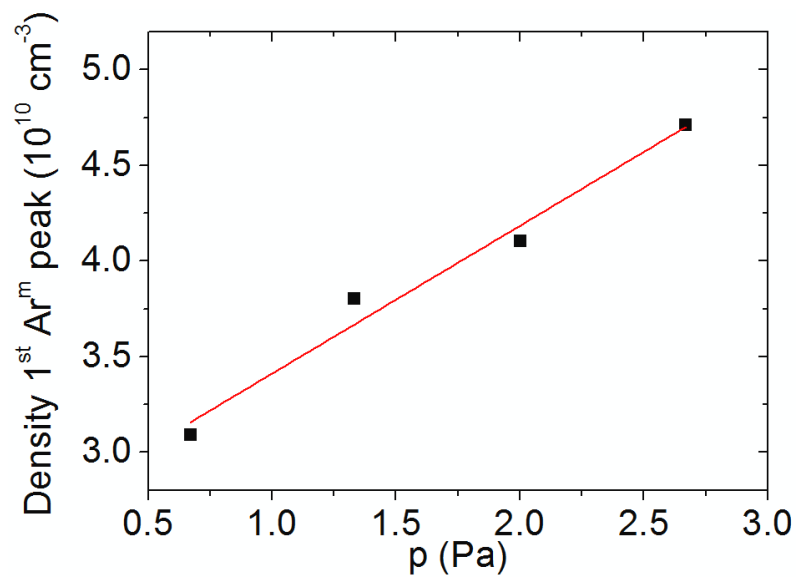

Figure 9. The density of the first $\mathrm{Ar}^{\mathrm{m}}$ peak plotted for different $\mathrm{Ar}$ pressures (0.67, 1.33, 2.00, and 2.67 Pa). The data is taken from Figure 8.

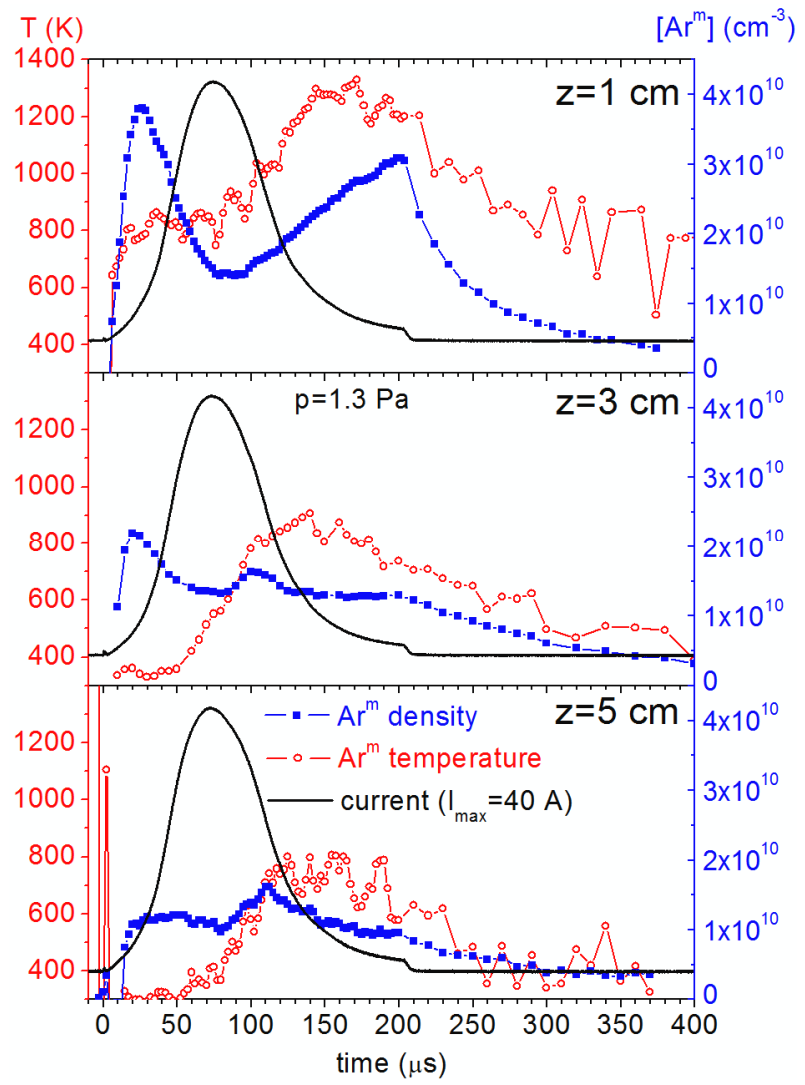

Figure 10. Time evolution of HiPIMS current, $\mathrm{Ar}^{\mathrm{m}}$ density, and $^{\mathrm{Am}}{ }^{\mathrm{m}}$ temperature for $40 \mathrm{~A}$ peak current, $200 \mu$ s pulse widths, 1.33 Pa pressure, measured at (top) $1 \mathrm{~cm}$ (middle) $3 \mathrm{~cm}$, and (bottom) $5 \mathrm{~cm}$ distance from the target. 

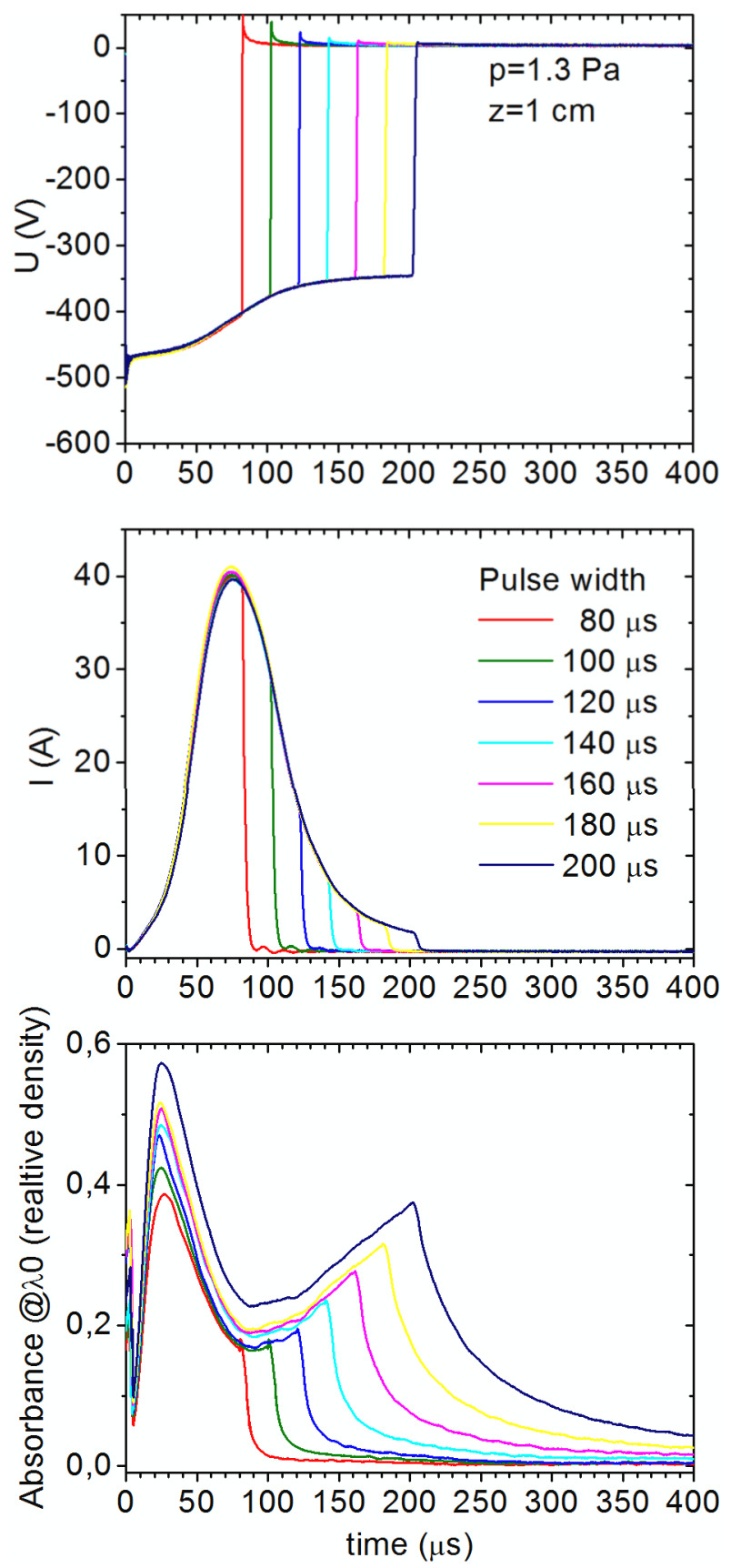

Figure 11. Time evolution of (top) HiPIMS voltage; (middle) HiPIMS current; (bottom) $\mathrm{Ar}^{\mathrm{m}}$ density; for $40 \mathrm{~A}$ maximum current, measured at $1 \mathrm{~cm}$ in front of the target for different pulse widths $(80,100,120$, $140,160,180,200 \mu \mathrm{s})$. 


\section{References}

[1] Helmersson U, Lattemann M, Bohlmark J, Ehiasarian A P and Gudmundsson J T 2006

\section{Thin Solid Films $\mathbf{5 1 3} 1$}

[2] Bohlmark J, Lattemann M, Gudmundsson J T, Ehiasarian A P, Aranda Gonzalvo Y, Brenning N and Helmersson U 2006 Thin Solid Films 5151522

[3] Lundin D, Larsson P, Wallin E, Lattemann M, Brenning N and Helmersson U 2008 Plasma Sources Sci. Technol. 17035021

[4] Samuelsson M, Lundin D, Jensen J, Raadu M A, Gudmundsson J T and Helmersson U 2010 Surf. Coat. Technol. 15591

[5] Alami J, Persson P O Å, Music D, Gudmundsson J T, Bohlmark J and Helmersson U 2005 J. Vac. Sci. Technol. A 23278

[6] Emmerlich J, Mráz S, Snyders R, Jiang K and Schneider J M 2008 Vacuum 82867

[7] Alami J, Sarakinos K, Mark G and Wuttig M 2006 Appl. Phys. Lett. 89, 154104

[8] Konstantinidis S, Dauchot J P, Ganciu M and Hecq M 2006 J. Appl. Phys. 99013307

[9] Lundin D, Brenning N, Jädernäs D, Larsson P, Wallin E, Lattemann M, Raadu M A and Helmersson U 2009 Plasma Sources Sci. Technol. 18045008

[10] Hoffman D W 1985 J. Vac. Sci. Technol. A 3561

[11] Rossnagel S M 1988 J. Vac. Sci. Technol. A 619

[12] Palmero A, Rudolph H and Habraken F H P M 2005 Appl. Phys. Lett. 87071501

[13] Palmero A, Rudolph H and Habraken F H P M 2006 Thin Solid Films 515631

[14] Kolev I and Bogaerts A 2008 J. Appl. Phys. 104093301

[15] Horwat D and Anders A 2010 J. Appl. Phys. 108123306

[16] Kadlec S 2007 Plasma Proc. Polym. 4 S419

17 Stancu G D, Ropcke J, Davies P B, 2005 J. Chem. Phys. 122014306 
[18] de Poucques L, Vitelaru C, Minea T M, Bretagne J and Popa G 2008 Europhys. Lett. 82 15002

[19] Beverini N, Genovesi G, Licchelli M, Maccarrone F, Marsili P and Strumia F 1998 Laser Phys. 8574

[20] Timmermans E A H, van de Sande M J and van der Mullen J J A M 2003 Plasma Sources Sci. Technol. 12324

[21] Vitelaru C, de Poucques L, Minea T M and Popa G 2011 J. Appl. Phys. 109053307

[22] Cazan R, Borcia G, Chiper A and Popa G 2008 Plasma Sources Sci. Technol.17 035020

[23] Stefanovic I, Sadeghi N and Winter J 2010 J. Phys. D. 43

152003

[24] Clarenbach B, Lorenz B, Kramer M and Sadeghi N 2003 Plasma Sources Sci. Technol. 12345

[25] Zybin A, Koch J, Wizemann H D, Franzke J and Niemax K 2005 Spectrochimica Acta Part B 601

[26] Galbacs G 2006 Appl. Spectrosc. Rev. 41259

[27] Demtröder W 2008 Laser Spectroscopy, Vol.2: Experimental Techniques (4th ed.)

(Berlin Heidelberg) Springer-Verlag

[28] Mitchell A and Zemansky M 1961 Resonance Radiation and Excited Atoms (Cambridge)

Cambridge University Press

[29] Hollas J M 2004 Modern Spectroscopy,(4th edition ) (Chichester) John Wiley \& Sons Ltd,

[30] Wolter M, Tung Do H, Steffen H and Hippler R 2005 J. Phys. D 382390

[31] Olejnicek J, Tung Do H, Hubicka Z, Hippler R and Jastrabik L 2006 Jpn. J. Appl. Phys. 458090 
[32] Gudmundsson J T, Sigurjonsson P, Larsson P, Lundin D and Helmersson U 2009 J. Appl. Phys. 105123302

[33] Poolcharuansin P and Bradley J W 2010 Plasma Sources Sci. Technol. 19025010

[34] Britun N and Han, J G 2008 Thin Solid Films 5166542

[35] Gudmundsson J T 2008 J. Phys.: Conf. Ser. 100082013

[36] Gudmundsson J T 2010 Vacuum 841360

[37] Sigurjonsson P 2008 Spatial and temporal variation of the plasma parameters in a high power impulse magnetron sputtering (HiPIMS) discharge (Reykjavik) Faculty of Engineering, University of Iceland 